\title{
Genotipificación de los factores de virulencia vacA y cagA de Helicobacter pylori en individuos de dos regiones de Colombia con riesgo opuesto de cáncer gástrico
}

\author{
Esperanza Trujillo', Teresa Martínez², María Mercedes Bravo \\ ${ }^{1}$ Grupo de Investigación en Cáncer y Agentes Infecciosos, Instituto Nacional de Cancerología, Bogotá, D.C., \\ Colombia \\ 2 Grupo de Investigación Epidemiológica del Cáncer, Instituto Nacional de Cancerología, Bogotá, D.C., Colombia \\ Institución donde se realizó en trabajo: Instituto Nacional de Cancerología, Bogotá, Colombia \\ Introducción. La prevalencia de infección por Helicobacter pylories alta en Colombia; en la zona andina \\ las tasas de cáncer gástrico son altas mientras que en las zonas costeras son bajas. Los genotipos de \\ $H$. pylori cagA positivo y vacA $s 1$ y $m 1$ se asocian con un mayor riesgo de cáncer gástrico. \\ Objetivo. Determinar las diferencias en las frecuencias de los genotipos de $H$. pylori asociados a \\ virulencia en dos regiones de Colombia con riesgo opuesto de cáncer gástrico. \\ Materiales y métodos. Se analizaron 401 biopsias del antro gástrico provenientes de 401 individuos \\ con diagnóstico de gastritis no atrófica, gastritis atrófica o metaplasia intestinal; 256 se obtuvieron en \\ la zona de alto riesgo (Tunja y Bogotá) y, 145, en la zona de bajo riesgo (Barranquilla, Santa Marta y \\ Cartagena). La genotipificación de los genes de virulencia cagA y vacA se hizo mediante reacción en \\ cadena de la polimerasa (PCR). \\ Resultados. No se observó diferencia en la frecuencia de infección por $H$. pylori entre las dos zonas \\ (77,3 Vs. 77,9 \%, p=no significativo, ns). La presencia de cagA fue mayor en la zona de bajo riesgo \\ (77,9 Vs. 69,2\%, p=ns). El alelo vacA s1 también fue más prevalente en la zona de bajo riesgo $(61,8$ \\ Vs. $72,0 \%, \mathrm{p}=\mathrm{ns})$. El alelo vacA $m 1$ presentó mayor prevalencia en la zona de alto riesgo (57,2 Vs. \\ $42,8 \%, \mathrm{p}=\mathrm{ns})$. La combinación cagA positivo $s 1 \mathrm{~m} 1$ también fue más frecuente en la zona de bajo \\ riesgo (48,9 Vs. 38,9\%, $\mathrm{p}=\mathrm{ns})$. \\ Conclusiones. Las diferencias en el riesgo de cáncer gástrico en estas dos zonas no pueden \\ explicarse por las diferencias en la prevalencia de infección por $H$. pylori o en la virulencia de las \\ cepas circulantes.
}

Palabras clave: Helicobacter pylori, genotipo, enfermedades gastrointestinales. doi: http://dx.doi.org/10.7705/biomedica.v34i4.2273

Genotyping of Helicobacter pylori virulence factors vacA and cagA in individuals from two regions in Colombia with opposing risk for gastric cancer

Introduction: The overall prevalence of Helicobacter pylori infection is high in Colombia; however, in the country's Andean region, gastric cancer rates far surpass those in coastal areas. Helicobacter pylori genotypes cagA positive and vacA s1 and $m 1$ are associated with an increased risk of gastric cancer. Objective: To compare the distribution of $H$. pylori genotypes associated with virulence in two regions in Colombia with opposing risk for gastric cancer.

Materials and methods: Four hundred and one gastric antral biopsies were obtained and analyzed from 401 individuals diagnosed with non-atrophic gastritis, atrophic gastritis and intestinal metaplasia: 256 came from the high-risk area cities of Tunja and Bogotá, and 145 from the low-risk area cities of Barranquilla, Santa Marta and Cartagena. Genotyping of virulence genes vacA and $\operatorname{cag} A$ was performed by PCR.

Results: No difference was observed in the frequency of $H$. pylori infection between the two areas (77.3\% vs $77.9 \%, p=$ non significant, ns). The presence of cagA was higher in the low-risk area $(77.9 \%$ vs. $69.2 \%, p=n s)$. The vacA s1 allele was also more prevalent in the low-risk area $(61.8 \%$ vs $72.0 \%$,

\footnotetext{
Contribución de los autores:

Teresa Martínez y María Mercedes Bravo: diseño del estudio

Esperanza Trujillo: realización de los ensayos

María Mercedes Bravo: elaboración del manuscrito

Todos los autores participaron en el análisis de los datos.
} 
$\mathrm{p}=\mathrm{ns})$. The vacA $m 1$ allele was more prevalent in the high-risk area ( $57.2 \%$ vs $42.8 \%, p=n s)$. The cagA positive $s 1 \mathrm{~m} 1$ combination was also more frequent in the low-risk area ( $48.9 \%$ vs $38.9 \%, p=n s)$.

Conclusions: The differences in the risk of gastric cancer in these two geographic areas cannot be explained by differences in the prevalence of infection by $\mathrm{H}$. pylori or by differences in the virulence of circulating strains.

Key words: Helicobacter pylori, genotype, gastrointestinal disease.

doi: http://dx.doi.org/10.7705/biomedica.v34i4.2273

El cáncer gástrico ocupa el quinto lugar en incidencia y el tercero en mortalidad a nivel mundial (1). En Colombia constituye la principal causa de muerte por cáncer (2). La infección con Helicobacter pylori se considera el factor de riesgo más importante para cáncer gástrico (3). H. pylori infecta a más del $50 \%$ de la población mundial (4). En todos los individuos infectados la bacteria causa gastritis crónica activa, que en 10 a $15 \%$ de los casos progresa a úlcera péptica, gastritis crónica atrófica, metaplasia intestinal, displasia y cáncer gástrico o hacia linfoma de tejido linfoide asociado a la mucosa (Mucosa-Associated Lymphoid Tissue, MALT) (5-7). Si bien el papel de la colonización persistente de la mucosa gástrica con $\mathrm{H}$. pylorien la patogénesis del cáncer gástrico, se ha confirmado en múltiples estudios de casos y controles (3) y en modelos animales (8), no se conocen los factores que determinan los resultados tan disímiles que puede tener la infección, ni por qué solo una minoría de individuos infectados desarrolla una enfermedad grave.

Varios genes de $H$. pylori se han asociado con la virulencia y la enfermedad gastroduodenal grave, entre los cuales el gen asociado a la citotoxina $(\operatorname{cag} A)$ y el gen de la citotoxina vacuolizante (vacA), se han estudiado ampliamente. El gen cagA es marcador de la presencia de un islote de patogenia (9), el cual codifica un sistema de secreción de tipo IV que transfiere la proteína CagA al interior de las células epiteliales (10). La infección con cepas positivas para cagA se asocia con un mayor riesgo de gastritis atrófica, úlcera péptica y cáncer gástrico (11-13). El gen vacA codifica una citotoxina vacuolizante y está presente en todos los aislamientos de $H$. pylori (13). Este gen contiene dos regiones variables: la región $s$, que

\section{Correspondencia:}

María Mercedes Bravo, Grupo de Investigación en Cáncer y Agentes Infecciosos, Instituto Nacional de Cancerología, Calle $1 \mathrm{~N}^{\circ}$ 9-85, Bogotá, D.C., Colombia

Teléfono: (571) 344 1111, extensión 4220

mbravo@cancer.gov.co

Recibido: 19/02/14; aceptado: 25/06/14 codifica el péptido señal y presenta dos variantes alélicas, $s 1$ y $s 2$, y la región $m$, o región media, que presenta las variantes alélicas $m 1$ y $m 2$. Los aislamientos de $H$. pylori con las combinaciones $s 1 m 1$ y $s 1 m 2$ producen, respectivamente, niveles altos y moderados de citotoxina, mientras que los aislamientos con la combinación s2m2 producen muy poca citotoxina o no la producen (14).

Aunque en Colombia la prevalencia de infección por $H$. pylori es alta en varias regiones $(15,16)$, existen acentuadas diferencias en la incidencia de cáncer gástrico entre las zonas montañosas de los Andes, con tasas muy altas, y las regiones costeras, con tasas muy bajas $(17,18)$. El propósito de este estudio fue determinar las frecuencias de los genotipos bacterianos cagA y vacA s1/s2 y $\mathrm{m1} /$ $m 2$ en dos regiones geográficas de Colombia con alta y baja incidencia de cáncer gástrico.

\section{Materiales y métodos \\ Población de estudio}

Los individuos incluidos en este estudio fueron reclutados en el marco del proyecto "Helicobacter pylori: prevalencia internacional, úlcera duodenal y neoplasia gástrica". Se incluyeron 401 pacientes con dispepsia que asistieron, entre febrero de 2000 y julio de 2008, a los servicios de gastroenterología de hospitales públicos y privados de las ciudades de Bogotá y Tunja (zona de alto riesgo) y de Barranquilla, Santa Marta y Cartagena (zona de bajo riesgo), y a quienes se les practicó una endoscopia de vías digestivas para el diagnóstico de enfermedad gastroduodenal. En la figura 1 se muestra la ubicación geográfica de las zonas de alto y bajo riesgo. Todos los individuos firmaron un consentimiento informado y el estudio fue aprobado por el Comité de Ética e Investigaciones del Instituto Nacional de Cancerología.

\section{Histopatología}

A todos los individuos se les tomaron 12 biopsias (5 del antro, 5 del cuerpo y 2 de la incisura angular). Tres biopsias de antro y tres de cuerpo se fijaron en formalina y se incluyeron en parafina, y una biopsia de antro y dos de cuerpo se almacenaron a $-70^{\circ} \mathrm{C}$ 


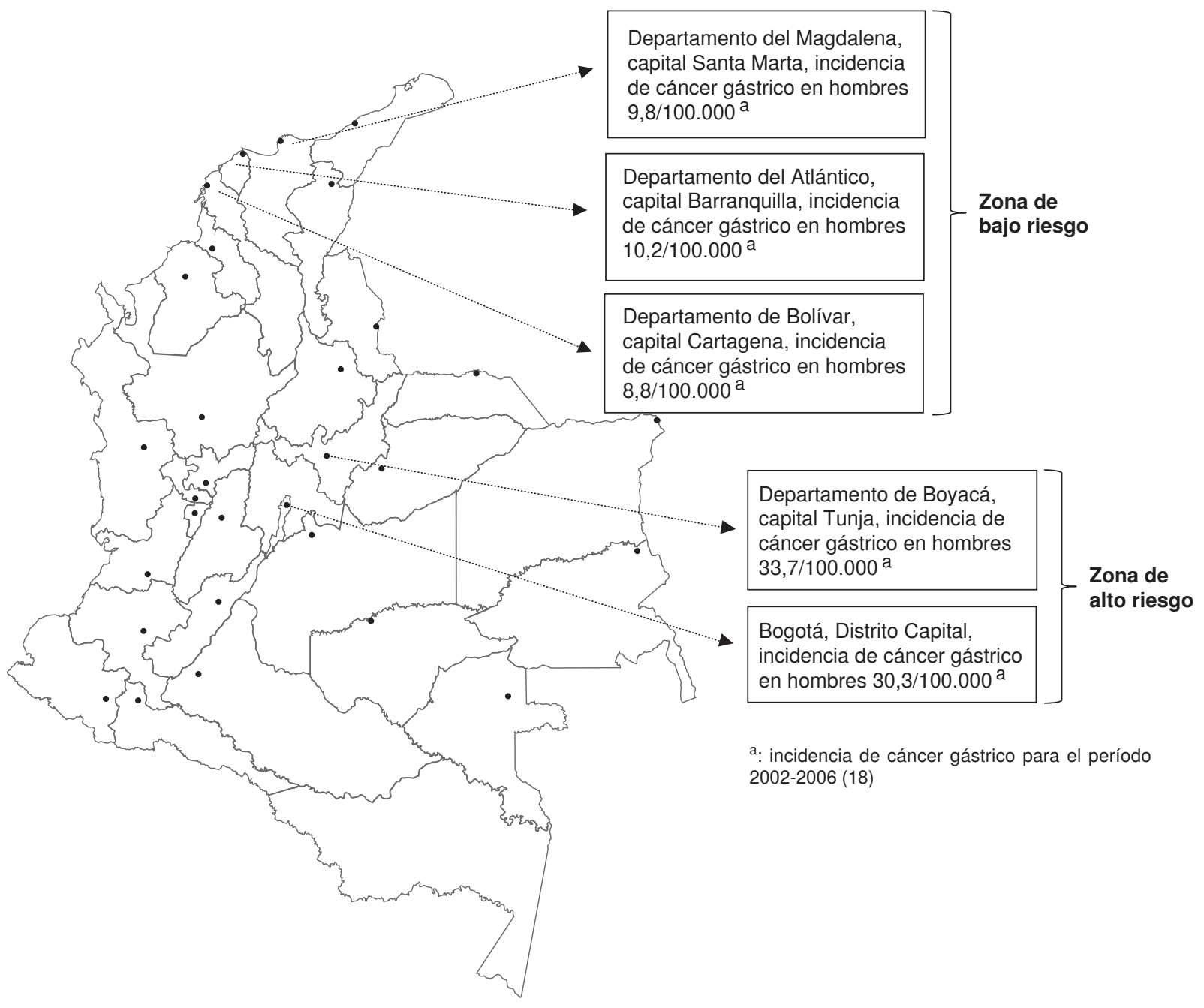

Figura 1. Localización geográfica de las zonas de alto y bajo riesgo de cáncer gástrico

en caldo de Brucella; las tres biopsias restantes se almacenaron a $-70{ }^{\circ} \mathrm{C}$ en seco. Para el estudio histopatológico se hicieron secciones de $3 \mu \mathrm{m}$ que luego se colorearon con hematoxilina y eosina. La clasificación histológica de las biopsias se ajustó a la versión revisada del sistema de Sidney en tres grupos de diagnóstico: gastritis crónica sin atrofia, gastritis crónica atrófica y gastritis crónica atrófica con metaplasia intestinal. La lesión más grave encontrada en una de las seis biopsias se empleó como diagnóstico global para cada paciente.

\section{Extracción de ADN de las biopsias gástricas}

Para la genotipificación de $H$. pylori se extrajo ADN total mediante tratamiento con proteinasa $\mathrm{Ka}$ partir de una biopsia de antro que estaba almacenada a $-70^{\circ} \mathrm{C}$ en caldo de Brucella. Brevemente expuesto, las biopsias se sumergieron durante dos horas a $56^{\circ} \mathrm{C}$ en $100 \mu$ de solución tampón de digestión (Tris-HCl
10 mM pH 8,0, KCl 50 mM, Tritón X-100 0,1 \% y 0,4 $\mathrm{mg}$ de proteinasa $\mathrm{K}$ ). El material digerido se calentó a $95{ }^{\circ} \mathrm{C}$ durante 15 minutos para desnaturalizar la proteinasa $\mathrm{K}$ y, luego de una centrifugación de 10 minutos a $10.000 \mathrm{rpm}$, el sobrenadante se utilizó para la reacción en cadena de la polimerasa (PCR).

\section{Análisis de vacA y cagA mediante PCR}

Los genes vacA y cagA se evaluaron mediante PCR según la metodología reportada por Yamaoka, et al.(19). Cada reacción de PCR contenía, en un volumen de $25 \mu \mathrm{l}$, TRIS-HCl $10 \mathrm{mM} \mathrm{pH} 8, \mathrm{KCl} 50$ $\mathrm{mM}, \mathrm{MgCl}_{2} 2,5 \mathrm{mM}$, dNTPs $200 \mu \mathrm{M}$, iniciadores (25 pM), $5 \mu \mathrm{l}$ del ADN extraído de las biopsias y $1 \mathrm{U}$ de polimerasa Taq (Promega, Madison, WI).

Las condiciones de la PCR incluyeron un paso inicial de desnaturalización a $94{ }^{\circ} \mathrm{C}$ seguido de 35 ciclos de un minuto a $94{ }^{\circ} \mathrm{C}$, un minuto a $52{ }^{\circ} \mathrm{C}$ y 
un minuto a $72{ }^{\circ} \mathrm{C}$, y un paso final de extensión a $72{ }^{\circ} \mathrm{C}$ durante 7 minutos. Los productos de la PCR se analizaron mediante electroforesis en geles de agarosa al 1,5 \% y tinción con bromuro de etidio. Como controles se utilizaron la cepa de referencia NCTC11638 con genotipo positivo para vacA s1m1 cagA y la cepa 3062 con genotipo negativo para vacA $s 2 m 2$ cagA obtenida en un estudio previo (16).

\section{Análisis estadístico}

Los datos se analizaron con el programa SPSS, versión 18. La prueba de ji al cuadrado y la prueba $\mathrm{T}$ se emplearon para determinar la significancia estadística de las diferencias en las frecuencias de los genotipos bacterianos y en la distribución de la edad entre las dos zonas de riesgo evaluadas.

\section{Resultados}

Se analizaron 401 biopsias gástricas provenientes de individuos de dos áreas geográficas de Colombia; 256 se obtuvieron en la zona de alto riesgo y, 145, en la zona de bajo riesgo.

Las características de los dos grupos se presentan en el cuadro 1. No se observaron diferencias significativas en el promedio de edad, la distribución de sexos fue significativamente diferente, $54 \%$ de los pacientes en la zona de alto riesgo y $35 \%$ en la zona de bajo riesgo eran hombres $(p<0,001)$.

La distribución de los diagnósticos histopatológicos fue significativamente diferente, ya que en la zona de alto riesgo se observó una mayor frecuencia de metaplasia intestinal mientras que en la zona de bajo riesgo la gastritis sin atrofia fue el diagnóstico más frecuente.

La frecuencia de la infección con $H$. pylori, determinada por la detección de, al menos, uno de los genes bacterianos evaluados, fue similar en las dos zonas: $77,3 \%$ en la zona de alto riesgo y $77,9 \%$ en la zona de bajo riesgo.

El gen cagA se detectó en 225 de las 311 biopsias positivas para infección con $H$. pylori $(72,3 \%)$. Los alelos $s 1$ y s2 se encontraron en 177 (56,9\%) y en $94(30,2 \%)$ de las biopsias positivas para infección con $H$. pylori, respectivamente; en 14 $(4,5 \%)$ biopsias se observaron los alelos $s 1$ y $s 2$ y en $26(8,4 \%)$ de ellas no se logró amplificar la región $s$. Los alelos $m 1$ y $m 2$ se detectaron en 146 $(46,9 \%)$ y $99(31,8 \%)$ de las biopsias positivas para infección con $H$. pylori, respectivamente. En seis $(2,1 \%)$ biopsias se observaron los alelos $m 1$ y $m 2$ y en 60 (19,3\%) de ellas no se logró amplificar la región $m$.
Cuadro 1. Características de los pacientes en las zonas de alto y bajo riesgo de cáncer gástrico

\begin{tabular}{|c|c|c|c|c|c|}
\hline & \multicolumn{2}{|c|}{$\begin{array}{c}\text { Zona de } \\
\text { alto riesgo } \\
n=256\end{array}$} & \multicolumn{2}{|c|}{$\begin{array}{c}\text { Zona de } \\
\text { bajo riesgo } \\
n=145\end{array}$} & $\mathbf{p}$ \\
\hline \multicolumn{6}{|l|}{ Edad (años) } \\
\hline Promedio (DE) & \multirow{2}{*}{\multicolumn{2}{|c|}{$\begin{array}{c}53,6(13,3) \\
(30-89)\end{array}$}} & \multirow{2}{*}{\multicolumn{2}{|c|}{$\begin{array}{c}48,3(13,1) \\
(30-85)\end{array}$}} & \\
\hline Rango & & & & & $0,001^{\mathrm{a}}$ \\
\hline \multicolumn{6}{|l|}{ Sexo } \\
\hline Masculino & 140 & $(54,7)$ & 52 & $(35,9)$ & \\
\hline Femenino & 116 & $(45,3)$ & 93 & $(64,1)$ & $0,000^{\mathrm{b}}$ \\
\hline \multicolumn{6}{|c|}{ Diagnóstico histopatológico } \\
\hline Gastritis & 94 & $(36,7)$ & 94 & $(64,8)$ & \\
\hline Gastritis atrófica & 57 & $(22,3)$ & 31 & $(21,4)$ & \\
\hline Metaplasia intestinal & 105 & $(41,0)$ & 20 & $(13,8)$ & $0,000^{\mathrm{b}}$ \\
\hline \multicolumn{6}{|l|}{ Infección con H. pylori } \\
\hline Positiva & 198 & $(77,3)$ & 113 & $(77,9)$ & \\
\hline Negativa & 58 & $(22,7)$ & 32 & $(22,1)$ & $n s^{b}$ \\
\hline
\end{tabular}

Cuadro 2. Distribución de los genotipos cagA y vacA para las dos áreas geográficas con riesgo opuesto

\begin{tabular}{|c|c|c|c|}
\hline & $\begin{array}{c}\text { Zona de } \\
\text { alto riesgo } \\
\text { n (\%) }\end{array}$ & $\begin{array}{c}\text { Zona de } \\
\text { bajo riesgo } \\
\text { n (\%) }\end{array}$ & $\mathbf{p}^{\mathrm{a}}$ \\
\hline \multicolumn{4}{|l|}{$\operatorname{cagA}$} \\
\hline cagA positivo & $137(69,2)$ & $88(77,9)$ & \\
\hline cagA negativo & $61(30,2)$ & $25(22,1)$ & ns \\
\hline \multicolumn{4}{|l|}{$\operatorname{vac} A s$} \\
\hline$s 1$ & $110(61,8)$ & $67(72,0)$ & \\
\hline s2 & $68(38,2)$ & $26(28,0)$ & ns \\
\hline \multicolumn{4}{|l|}{ vacAm } \\
\hline$m 1$ & $99(57,2)$ & $74(42,8)$ & \\
\hline$m 2$ & $47(65,3)$ & $25(34,7)$ & ns \\
\hline \multicolumn{4}{|l|}{$\begin{array}{l}\text { Combinación cagA } \\
\text { positivo /s1m1 Vs. otras }\end{array}$} \\
\hline cagA positivo/s $1 \mathrm{~m} 1$ & $92(48,9)$ & $41(38,9)$ & \\
\hline Otros & $96(51,1)$ & $65(61,3)$ & ns \\
\hline
\end{tabular}

a: ji al cuadrado; ns: no significativo

La distribución de los genotipos de cagA y vacA en las dos áreas geográficas con riesgo opuesto para cáncer gástrico, se presenta en el cuadro 2; se excluyeron los casos en que se encontraron infecciones mixtas, o en los que no fue posible amplificar la región $m$ o $s$ del gen vacA. La prevalencia de cepas con cagA positivo fue mayor en la zona de bajo riesgo; igualmente, el alelo $s 1$ se detectó con mayor frecuencia en esta zona. El alelo $m 1$ fue más prevalente en la zona de alto riesgo. Se observó una mayor frecuencia de cepas cagA positivo $s 1 \mathrm{~m} 1$ en la zona de alto riesgo. Estas diferencias no fueron significativas. Los genotipos cagA y vacA s1 se correlacionaron significativamente en ambas poblaciones. En la zona de alto riesgo, 107 de las 119 cepas con cagA 
positivo fueron positivas para $s 1(p<0,001)$ y, en la de bajo riesgo, 64 de las 69 cepas con cagA positivo lo fueron también para $s 1(p<0,0001)$.

En la figura 2 se presenta la distribución de los diagnósticos histopatológicos y los genotipos de $\mathrm{H}$. pylori según la zona de riesgo. En las dos zonas de riesgo se observó un incremento en la frecuencia de $\operatorname{cag} A$ y de los alelos $s 1$ y $m 1$ a medida que aumentaba el grado de la lesión; en la zona de bajo riesgo estas diferencias no fueron significativas. En la zona de alto riesgo se observó una frecuencia de $\operatorname{cag} A, s 1$ y $m 1$ significativamente mayor en los casos de metaplasia intestinal, comparados con los de gastritis $(p<0,0001)$.

\section{Discusión}

Al igual que en otros países latinoamericanos, en Colombia se observan altas tasas de mortalidad por cáncer gástrico en las zonas montañosas de los Andes, mientras que en las zonas costeras

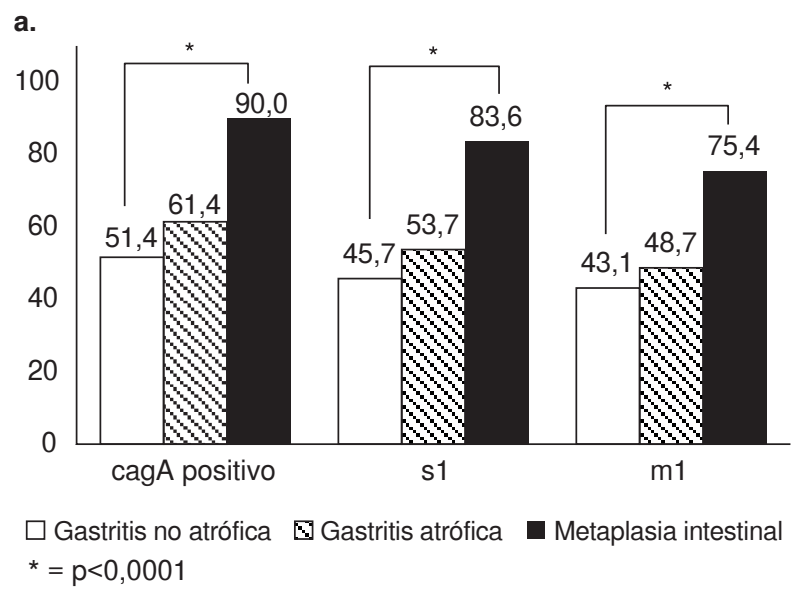

b.

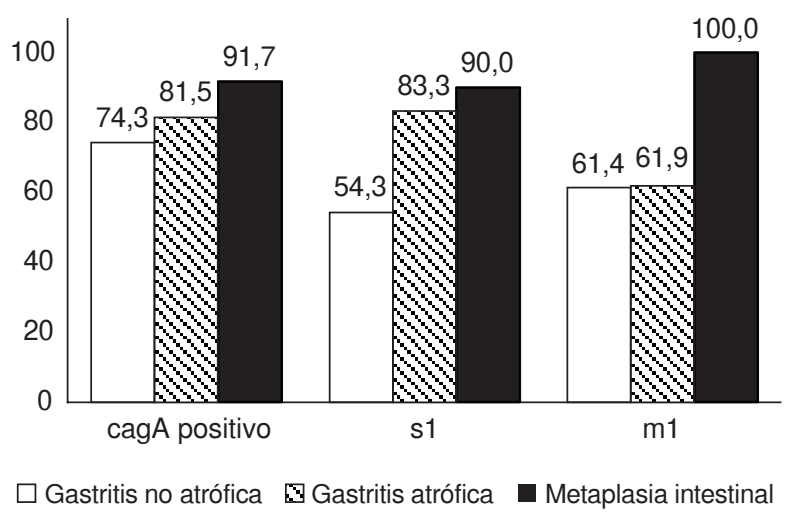

Figura 2. a. Distribución de los diagnósticos histopatológicos y los genotipos de Helicobacter pylori en la zona de alto riesgo. b. Distribución de los diagnósticos histopatológicos y los genotipos de Helicobacter pylori en la zona de bajo riesgo del Pacífico y el Atlántico se observan tasas de mortalidad mucho más bajas (17). En este trabajo se investigó la presencia de H. pylori y la distribución de los genes de virulencia vacA y cagA en individuos provenientes de la zona andina (Tunja) y de la zona atlántica (Barranquilla, y Cartagena), dos zonas geográficas con riesgo opuesto de cáncer gástrico, es decir, alto en la zona andina y bajo en la zona atlántica. En ambas regiones se han registrado altas tasas de infección por $H$. pylori. Bravo, et al. (15), y Sicinschi, et al. (19), reportaron altas tasas de infección por $H$. pylori también en la región montañosa del sur de Colombia (Pasto y Túquerres) y en Tumaco, en la Costa Pacífica, zonas con riesgo opuesto de cáncer gástrico. Las diferencias en las tasas de infección no explican las diferencias en la incidencia del cáncer gástrico entre las regiones.

En contraste con la zona sur del país, donde se reportaron frecuencias significativamente más altas de los alelos $s 1$ y $m 1$ y de la combinación $s 1 m 1$, así como la tendencia a una mayor frecuencia de cepas positivas para $\operatorname{cag} A$ en la zona montañosa de alto riesgo (20), en el presente estudio no se encontraron diferencias significativas en la distribución de los alelos cagA y vacA $s$ y $m$ en las zonas estudiadas, aunque, sorpresivamente, en la zona de la Costa Atlántica las frecuencias de los alelos de riesgo $s 1$ y $m 1$ y de cagA fueron mayores que en la zona andina, hecho llamativo considerando que la gastritis fue el diagnóstico más frecuente en la zona de bajo riesgo. La frecuencia de cepas positivas para cagA $s 1 m 1$ fue mayor en la zona de alto riesgo, aunque la diferencia no fue significativa.

Al evaluar la distribución de los diagnósticos histopatológicos y de los genotipos de H. pylori en las dos áreas de riesgo, se encontró que, si bien la frecuencia de los alelos $s 1, m 1$ y de cagA fue mayor en ambas zonas a medida que avanzaba la lesión, solo fue significativa en la zona de alto riesgo al comparar la gastritis y la metaplasia intestinal. La alta frecuencia de estos alelos en la gastritis observada en la zona de bajo riesgo no permitió detectar diferencias significativas con lesiones de mayor grado.

En este estudio el principal hallazgo fue el hecho de que las diferencias en la incidencia entre las dos zonas con riesgo opuesto para cáncer gástrico no se explican por una mayor frecuencia de la infección con $H$. pylori, ni por la presencia del gen de virulencia cagA o de los alelos de virulencia s1 y $m 1$. 
En un estudio reciente realizado en Túquerres, zona montañosa de alto riesgo al sur de Colombia, y en Tumaco, población de la costa pacífica con bajo riesgo de cáncer gástrico, Kodaman, et al., (21) reportaron que en los aislamientos de $H$. pylori de la zona de bajo riesgo predominó el ancestro africano, mientras que en los de la zona de alto riesgo predominó el ancestro europeo. Por otro lado, se observó el predominio del ancestro africano en los individuos infectados de la costa y del ancestro amerindio en los individuos infectados de la zona de montaña.

En dicho estudio se observó que la interacción entre los orígenes ancestrales del huésped y el patógeno explicaba la mayor gravedad de las lesiones gástricas en la zona de alto riesgo, y, en particular, que la infección por H. pylori en personas con ancestro africano era relativamente benigna, en tanto que podía ser mucho más grave entre individuos con ancestro amerindio. Probablemente, las variables clave en cuanto al riesgo son los patrones genómicos de variación en el patógeno y el huésped, los cuales interactúan y permiten predecir un fenotipo clínico.

Si bien estos investigadores observaron una asociación entre la zona de residencia y el grado histopatológico, así como una mayor frecuencia de lesiones avanzadas en la zona montañosa, esta asociación perdió significancia al considerar los ancestros del huésped, lo que indica que no son la localización ni la altitud las que explican la discrepancia en el riesgo.

En un futuro cercano, el propósito de los autores es analizar los orígenes ancestrales de la población estudiada en este trabajo, así como en los aislamientos de $\mathrm{H}$. pylori, para evaluar esta importante asociación entre los ancestros del patógeno y del huésped, y explicar las diferencias entre estas dos zonas en lo concerniente al riesgo.

\section{Conflicto de intereses}

Los autores declaran no tener ningún conflicto de intereses.

\section{Financiación}

Este proyecto fue financiado por el Instituto Nacional de Cancerología a través de los recursos de inversión asignados por la Nación en el rubro 41030310-4.

\section{Referencias}

1. International Agency for Research on Cancer. Globocan 2012. Fecha de consulta: 12 de junio de 2014. Disponible en: http://globocan.iarc.fr/Pages/fact_sheets_cancer.aspx.
2. Piñeros $\mathbf{M}$, Gamboa $\mathbf{O}$, Hernández-Suárez G, Pardo $\mathbf{C}$, Bray F. Patterns and trends in cancer mortality in Colombia 1984-2008. Cancer Epidemiol. 2013;37:233-9. http://dx.doi. org/10.1016/j.canep.2013.02.003

3. Helicobacter and Cancer Collaborative Group. Gastric cancer and Helicobacter pylori: A combined analysis of 12 case control studies nested within prospective cohorts. Gut. 2001;49:347-53. http://dx.doi.org/10.1136/gut.49.3.347

4. Suerbaum S, Michetti P. Helicobacter pylori infection. N Engl J Med. 2002;347:1175-86. http://dx.doi.org/10.1056/ NEJMra020542

5. Peek RM Jr, Blaser MJ. Helicobacter pylori and gastrointestinal tract adenocarcinomas. Nat Rev Cancer. 2002;2:2837. http://dx.doi.org/10.1038/nrc703

6. Blaser MJ, Berg DE. Helicobacter pylori genetic diversity and risk of human disease. J Clin Invest. 2001;107:767-73. http://dx.doi.org/10.1172/JCl12672

7. Israel DA, Peek RM. Pathogenesis of Helicobacter pyloriinduced gastric inflammation. Aliment Pharmacol Ther. 2001;15:1271-90. http://dx.doi.org/10.1046/j.1365-2036. 2001.01052.x

8. Honda S, Fujioka T, Tokieda M, Satoh R, Nishizono A, Nasu M. Development of Helicobacter pylori-induced gastric carcinoma in Mongolian gerbils. Cancer Res. 1998; 58:4255-9.

9. Censini S, Lange C, Xiang Z, Crabtree JE, Ghiara P, Borodovsky $\mathbf{M}$, et al. cag, a pathogenicity island of Helicobacter pylori, encodes type I-specific and diseaseassociated virulence factors. Proc Natl Acad Sci USA. 1996;93:14648-53.

10. Akopyants NS, Clifton SW, Kersulyte D, Crabtree JE, Youree BE, Reece CA, et al. Analyses of the cag pathogenicity island of Helicobacter pylori. Mol Microbiol. 1998;28:37-53. http://dx.doi.org/10.1046/j.1365-2958.1998. 00770.x

11. Blaser MJ, Pérez-Pérez GI, Kleanthous H, Cover TL, Peek RM, Chyou PH, et al. Infection with Helicobacter pylori strains possessing cagA is associated with an increased risk of developing adenocarcinoma of the stomach. Cancer Res. 1995;55:2111-5.

12. Kuipers EJ, Pérez-Pérez GI, Meuwissen SG, Blaser MJ. Helicobacter pylori and atrophic gastritis: Importance of the cagA status.J Natl Cancer Inst. 1995;87:1777-80. http://dx. doi.org/10.1093/jnci/87.23.1777

13. Phadnis SH, Ilver D, Janzon L, Normark S, Westblom TU. Pathological significance and molecular characterization of the vacuolating toxin gene of Helicobacter pylori. Infect Immun. 1994;62:1557-65.

14. Atherton JC, Cao P, Peek RM Jr, Tummuru MK, Blaser MJ, Cover TL. Mosaicism in vacuolating cytotoxin alleles of Helicobacter pylori. Association of specific vacA types with cytotoxin production and peptic ulceration. J Biol Chem. 1995;270:17771-7. http://dx.doi.org/10.1074/jbc.270. 30.17771

15. Bravo LE, van Doom LJ, Realpe JL, Correa P. Virulenceassociated genotypes of Helicobacter pylori: Do they explain the African enigma? Am J Gastroenterol. 2002; 97:2839-42. http://dx.doi.org/10.1111/j.1572-0241.2002.07031.x 
16. Quiroga AJ, Cittelly DM, Bravo MM. Frecuencia de los genotipos babA2, oipA y cagE de Helicobacter pylori en pacientes colombianos con enfermedades gastroduodenales. Biomédica. 2005;25:325-34. http://dx.doi.org/10.7705/ biomedica.v25i3.1357

17. Torres J, Correa $\mathbf{P}$, Ferreccio $\mathbf{C}$, Hernández-Suárez G, Herrero R, Cavazza-Porro M, et al. Gastric cancer incidence and mortality is associated with altitude in the mountainous regions of Pacific Latin America. Cancer Causes Control. 2013;24:249-56. http://dx.doi.org/10.1007/ s10552-012-0114-8

18. Pardo C, Cendales R. Incidencia estimada y mortalidad por cáncer en Colombia, 2002-2006. Bogotá, D.C: Instituto Nacional de Cancerología; 2010.

19. Yamaoka Y, Kodama T, Kita M, Imanishi J, Kashima K, Graham DY. Relationship of vacA genotypes of Helicobacter pylori to cagA status, cytotoxin production, and clinical outcome. Helicobacter.1998;3:241-53. http://dx.doi.org/10. 1046/j.1523-5378.1998.08056.x

20. Sicinschi LA, Correa P, Peek RM Jr, Camargo MC, Delgado A, Piazuelo MB, et al. Helicobacter pylori genotyping and sequencing using paraffin-embedded biopsies from residents of Colombian areas with contrasting gastric cancer risks. Helicobacter. 2008;13:135-45. http://dx. doi.org/10.1111/j.1523-5378.2008.00554.x

21. Kodaman N, Pazos A, Schneider BG, Piazuelo MB, Mera R, Sobota RS, et al. Human and Helicobacter pylori coevolution shapes the risk of gastric disease. Proc Natl Acad Sci USA. 2014;111:1455-60. http://dx.doi.org/10.1073/ pnas.1318093111 